\title{
ESPECIES DEL GENERO BOLETUS de la Sierra de las Cruces y del Desierto de los Leones, D. F."1
}

Por Monserrat Gispert de Imaz.

Los ejemplares que sirvieron para el presente estudio fueron colectados en la sierra de Las Cruces a una altitud de unos $3,200 \mathrm{~m}$. (límite del D. F. y el Estado de México) y en el Desierto de Los Leones, a una altitud media de $3,200 \mathrm{~m}$. (Distrito Federal). Ambas son regiones de clima montañoso, con atmósfera muy húmeda durante la estación de lluvias (aproximadamente de mayo a noviembre con una precipitación de 700-800 mm.). Las capas de tierra vegetal son bastante profundas, llegando hasta $2 \mathrm{~m}$; y los diversos detritos vegetales acumulados forman un terreno rico muy apropiado para el crecimiento de árboles y arbustos. El subsuelo es rocoso e impermeable.

\section{CARACTERES USADOS EN EL ESTUDIO \\ DEL GENERO BOLETUS}

1) Forma y tamaño.-Son importantes estos caracteres tanto para el hongo en general como para cada una de sus partes.

2) Superficie y estructura.-Es importante la superficie del píleo, que puede ser lisa, escamosa, granular, etc. Tiene importancia la cualidad viscosa o seca del píleo. La presencia o ausencia de reticulación en el estípite; éste puede presentar un anillo en algunas especies. Los tricodermios de la cutícula del píleo pueden encontrarse entrelazados, formando racimos, o sueltos.

3) Color.-Se ha tenido en cuenta la coloración del píleo, del estípite, de los poros y de las esporas. El color de estas últimas se puede obtener por impresión (en papel celofán o en papel blanco). Las coloraciones que toman las esporas poniéndolas en diferentes reactivos es también un carácter taxonómico de gran interés. Otro dato importante es el de las coloraciones (azules, lilas, verdosas, etc.) que en algunas especies se forman en la carne, los tubos, o los poros en contacto con el aire.

4) Esporas.-La forma, el tamaño y las características de las paredes de las esporas (lisas o estriadas), así como la presencia o ausencia de poro en las mismas, son datos muy importantes para la clasificación de las especies.

1) Presentado como tesis profesional para optar al título de Biólogo en la Facultad de Ciencias, U.N.A.M.

Gispert de Imaz M. 1958. Especies del género Boletus de la Sierra de las Cruces y del Desierto de los Leones, D.F. Boletín de la Sociedad Botánica de México 22: 28-40. 
5) Cistidios.-Aunque los cistidios son estructuras secundarias, tienen importancia debido a que son característicos para cada especie. En el género Boletus, los pleurocistidios y las queilocistidios son muy semejantes, sólo difieren por su posición; los primeros se encuentran en toda la superficie de los tubos; los segundos en el borde de los mismos, al nivel de los poros.

6) Tubos y poros. La forma, color y tamaño de los poros y de los tubos, así como la relación de los últimos con respecto al estípite, son datos indispensables. Los tubos pueden ser adnatos, decurrentes, libres, etc.

7) Olor y sabor.-Cuando se tiene el hongo fresco es conveniente anotar su olor y su sabor.

Con el hongo fresco se deben anotar los colores del píleo y el estípite, así como también obtener las impresiones de las esporas. Con el ejemplar fresco se deben observar los cambios de color en la carne y en los tubos.

Hay dos maneras de conservar los hongos: en alcohol (de 70-90) cuya desventaja es la pérdida de color; o bien secándolos en parrillas especiales, forma en la cual el hongo conserva una apariencia casi natural y por ello este es el método que generalmente se usa.

Para obtener las esporas de ejemplares conservados por desecación $o$ en alcohol, se toman de los tubos y se maceran en el portaobjetos. Las substancias usadas para la observación de las esporas fueron: $\mathrm{KOH}$ al $3 \%$, cloral lactofenol de Amann y solución de Lugol. Las observaciones de los cistidios se hicieron en cortes logrados con microtomo de mano, utilizando médula de saúco para sostener el material en estudio.

En el transcurso de este trabajo se tuvo la oportunidad de estudiar doce especies del género Boletus. Boletus spp., Boletus turbinatus, Boletus subtomentosus, Boletus pulveruientus, Boletus porphyrosporus, Boletus placidus, Boletus luridus, Boletus edulis, Boletus chrysenteron, Boletus calopus, Boletus brevipes.

Dentro de este género existen especies comestibles y no comestibles y aún reputadas como venenosas. La más comúnmente usada en la alimentación es Boletus edulis, conocida vulgarmente con los nombres de "pambazo" o "cemita", que se prepara en guisos populares locales condimentados con epazote (Chenopodium ambrosioides), o bien simplemente asados sobre el comai (a la plancha), aparte de que son también preparados según las fórmulas de la cocina internacional.

Entre las especies sospechosas de ser tóxicas aparecieron en nuestro material Boletus luridus y Boletus calopus.

Como signos para el reconocimiento de las especies comestibles de Boletus pueden admitirse los siguientes enumerados por Thomas (1948): "no tornarse azul la carne al ser cortada o rota, no tener poros de color rojo o anaranjado y finalmente carecer de sabor amargo o fuerce". 


\section{DESCRIPCION DE LAS ESPECIES}

Boletus brevipes Peck.

Píleo de 4 a $9 \mathrm{~cm}$. de ancho, convexo, con márgenes ligeramente lobulados, superficie glutinosa o viscosa y color moreno rojizo. Carne blanda, blanca cuando el ejemplar es joven, volviéndose amarilla con la edad; al contacto del aire no cambia de color; sabor y olor no especiales.

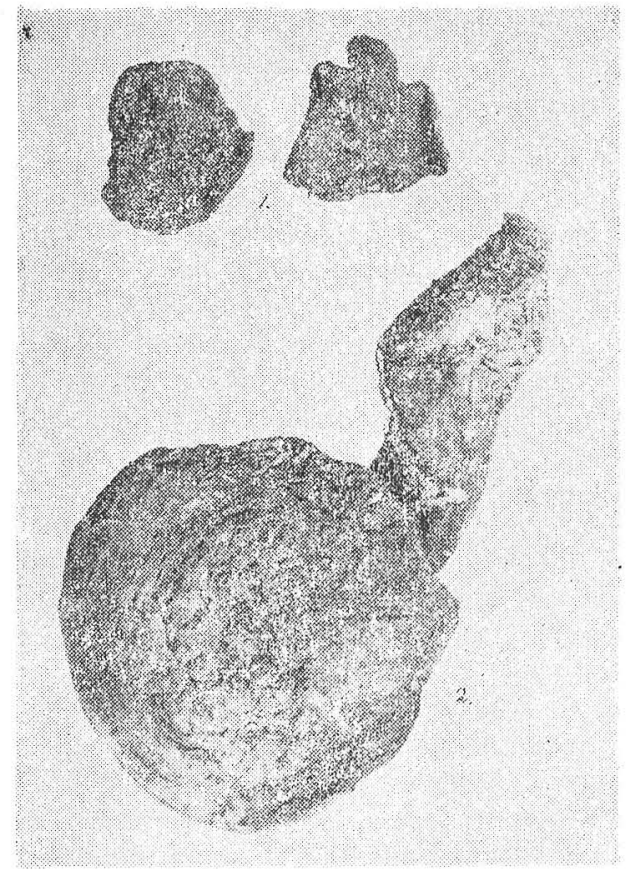

BOLETUS BREVIPES, vista dorsal y ventral; 2) BOLETUS CALOPUS, vista dorsal.

Tubos pequeños, adnatos, subdecurrentes, de 3 a $8 \mathrm{~mm}$. de profundidad, color amarillo, volviéndose más obscuros con la edad; no cambian de color al contacto del aire ni con el frotamiento.

Estípite corto, de 2 a $4 \mathrm{~cm}$. de largo por 1 a 2 de ancho, fuerte, sólido, moreno claro en la base y amarillo en el ápice.

Esporas de color moreno claro; en $\mathrm{KOH}$ presentan un color amarillo pálido; lisas, miden $9-10 \times 3$ micras. Pleutocistidios en racimos con par- 
tículas morenas alrededor, de un color moreno en el interior, de 30-50 x 6-7 micras, cilíndricas o en forma de clava, muchas veces hialinas; queilocistidios semejantes a los pleurocistidios o un poco más alargadas, con más aspecto de clava.

Habitat. En lugares musgosos; son comunes durante la temporada de lluvias.

Localidad: Sierra de Las Cruces.

Observación. Los ejemplares del Estado de Michigan (EE.UU.) al ser comparados con los de la Sierra de Las Cruces (México) presentaron solamente una pequeña diferencia que fué el menor tamaño de las esporas, de 7-8.5 x 2.8-3 micras, para el ejemplar norteamericano. Esta pequeña diferencia es debida seguramente a la altura y configuración del terreno que exploramos.

\section{Boletus calopus Fries}

Píleo de 5 a $11 \mathrm{~cm}$. de ancho, convexo, volviéndose plano con la edad; el margen a veces encorvado hacia adentro; superficie seca, estriada cuando joven; puede también presentar un aspecto areolado, volviéndose con la edad lisa o subtomentosa; de color amarillo moreno. Carne de 4-5 mm., gruesa, firme y que al contacto del aire toma un color azul intenso.

Tubos de 8-9 mm. de profundidad, de color amarillo pálido en los ejemplares jóvenes, volviéndose amarillo azuloso con la edad; son adnatos, pero forman líneas decurrentes sobre el estípite. Poros de color amarillo canario, ligeramente anaranjados, pequeños, primeramente redondos y angulares en la madurez; al cortarlos toman un color azul intenso.

Estípite de 7.5-12.5 cm. de largo por $1.5-3 \mathrm{~cm}$. de ancho en el ápice y de $2.5-4.5 \mathrm{~cm}$. en la base, grueso, sólido; carne amarilla que se vuelve instantáneamente azul cuando se corta; en la parte superior presenta una fina reticulación y una intensa tonalidad rojiza; la parte inferior es lisa, de color amarillo o amarillo anaranjado.

Esporas de 13-16 x 5-6 micras, lisas; paredes gruesas, color ocre pálido en $\mathrm{KOH}$. La cutícula del píleo morena pálida en $\mathrm{KOH}$, homogénea. Las hifas entretejidas en forma compacta; las paredes de las hifas con pálidas incrustaciones de pigmento.

Habit. Solitario o gregario, colectado en bosque de coníferas.

Localidad: Sierra de Las Cruces.

Venenoso. Se dice que después de cierto tratamiento se puede comer.

Observación. Al compararlos con ejemplares de Oregon (EE. UU.) se observó una pequeña diferencia en las medidas de las esporas, las cuales eran de 13-19 x 5-5.5 micras para los ejemplares estadounidenses. 


\section{Boletus chrysenteron Bull.}

Píleo de 8-13 $\mathrm{cm}$. de ancho, convexo, el margen ligeramente encorvado, con la superficie seca y de apariencia escamosa, de color moreno obscuro

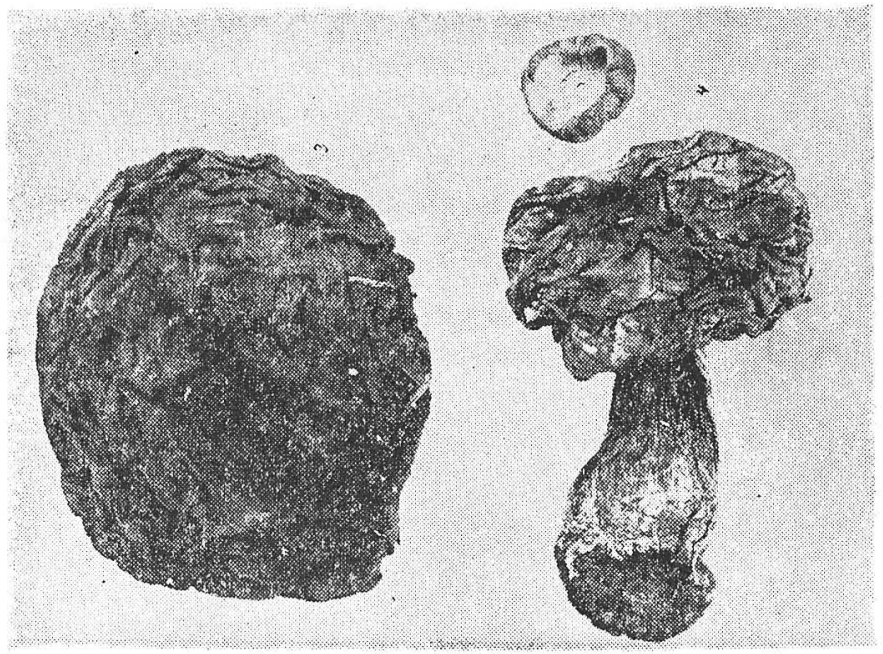

3, BOLETUS CHRYSENTERON, vista dorsal; BOLETUS EDULIS, vista dorsal y ventral.

en el centro y castaño claro en el borde, volviéndose más obscuro con la madurez. Carne de $8 \mathrm{~mm}$. de grosor, de color amarillo muy pálido, casi blanco, con una franja rosa; toma color azul cuando se corta; sabor ligeramente ácido.

Tubos de $10 \mathrm{~cm}$. de longitud, de color amarillo pálido, cambiando con la edad a amarillo verdoso y comprimidos alrededor del estípite. Poros angulares, de color amarillo olivo en los ejemplares jóvenes y olivo moreno en los maduros; al contacto del aire toman un color azul.

Estípite de 5-15 cm. de largo por $2-3 \mathrm{~cm}$. de ancho, grueso, sólido, de superficie rugosa, moreno grisáceo en la base y rojizo en el ápice.

Esporas de 13-16 x 5-6 micras, de color moreno oliváceo, lisas y de paredes gruesas. Cutícula del píleo con tricodermios entrelazados. Las paredes de las hifas presentan incrustaciones y las células terminales se encuentran ensanchadas; color ocre en $\mathrm{KOH}$. 
Habit. Gregarios, colectados en bosques de pino y oyamel. Localidad. Sierra de Las Cruces.

Comestible. (Aunque muchos autores tienen dudas acerca de ello).

Observación. En los ejemplares estudiados de Oregon (EE. UU.) las esporas miden 11-15 x 5-6 micras, o sea que la longitud es ligeramente inferior con respecto a los ejemplares mexicanos.

\section{Boletus edulis Bull.}

Píleo de 8-13 cm. de ancho, convexo, de superficie seca, viscosa en la humedad, bastante desigual, pues unas veces es lisa y otras rugosa, picada, etc.; color variable, moreno obseuro en el centro y moreno claro, casi amarillo, en la parte periférica; otros ejemplares son moreno pálidos, casi amarillos, y con áreas moreno obscuras. Carne de $1.5-3.5 \mathrm{~cm}$. de espesor, fuerte, volviéndose blanda con la edad; al contacto del aire no cambia de color, y únicamente cerca de la cutícula toma un color lila claro o moreno pálido; el color de la carne es blanquizco o amarillo muy claro; olor agradable, algunas veces con sabor a nuez.

Tubos adnatos, pero deprimidos, de $1.5-2.5 \mathrm{~cm}$. de profundidad, obliterados cuando jóvenes. Poros pequeños redondos, miden cerca de $2-3 \mathrm{~cm}$. por par; en los ejemplares jóvenes los tubos son blancos y se vuelven con el tiempo amarillo verdosos y finalmente moreno claros.

Estípite de $10.15 \mathrm{~cm}$. de longitud por 2-4 cm. de espesor en el ápice en ejemplares maduros, pero en los jóvenes mide alrededor de 3-4 cm. de espesor en el ápice y de 7-8 cm. en la base y es bulboso; siempre es sólido; ligeramente reticulado, estriado en la porción inferior, de color blanco con tonalidades morenas y amarillas.

Esporas de 17-22 x 5-6 micras, de color leonado pálido en $\mathrm{KOH}$, lisas, subfusiformes, de paredes gruesas.

Habitat. Gregario. Bajo coníferas en tierra con musgo; abundante. Localidad. Sierra de Las Cruces.

Nombre vulgar. Pambazo o cemita.

Observación. Las esporas de Boletus edulis que mencionan diferentes autores tienen, sin excepción, un tamaño inferior a 20 micras; al estudiar las esporas de Boletus edulis de Idaho (EE. UU.), se pudo comprobar que medían alrededor de 17-20 x 5-6 micras, que es el tamaño aproximado de las esporas de los ejemplares colectados en México. Esto seguramente es debido a que ambos ejemplares (el de la Sierra de Las Cruces $y$ el de Idaho) fueron encontrados en lugares montañosos. 


\section{Boletus luridus Shaeffer.}

Píleo de 3.5-8 cm. de ancho, convexo, de superficie seca, color moreno obscuro en los ejemplares jóvenes, en los maduros el color cambia, siendo moreno rojizo en la parte superior y amarillo anaranjado en el margen. Carne firme, gruesa, blanca grisácea en los ejemplares jóvenes, amarilla rojiza en los maduros; al contacto del aire toma un color azul intenso; sabor suave y olor no especial.

Tubos libres, de 1-2 cm. de profundidad; color amarillo, volviéndose instantáneamente azul intenso cuando se cortan. Poros redondos, pequenos, de color rojo ladrillo.

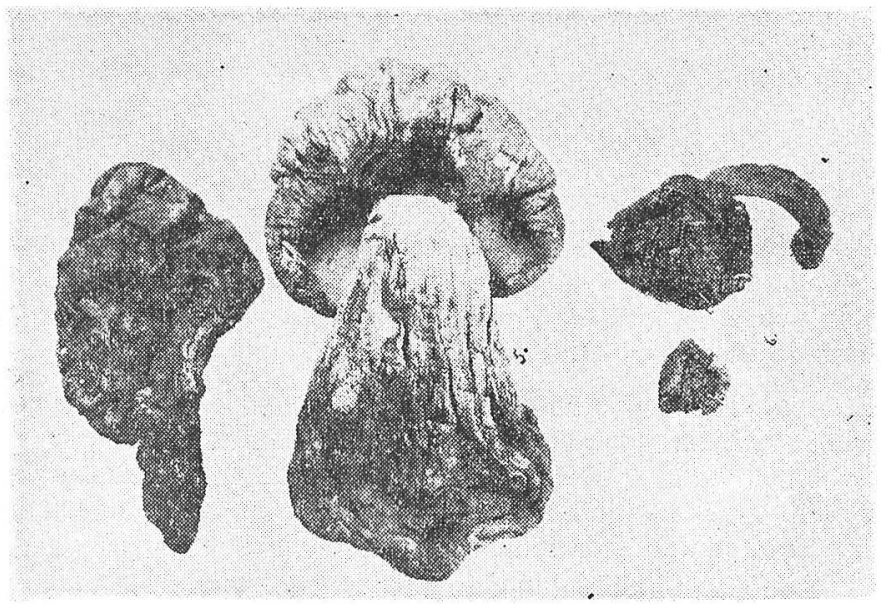

5, BOLETUS LURIDUS, vista dorsal y ventral; 6, BOLETUS PLACIDUS, vista ventral y dorsal.

Estípite de 4-9 cm. de largo x $1.5 \mathrm{~cm}$. de ancho en el ápice y de 2$2.5 \mathrm{~cm}$. de ancho en la base, sólido; la porción apical grandemente reticulada con estrías rojizas; bulboso en los ejemplares jóvenes; en los ejemplares maduros el color varía, siendo amarillo de huevo en la parte superior y moreno rojizo en la base.

Esporas de 11.7-17 x 5.5-7 micras, lisas, presentando un color ocre en KOH. La cutícula del píleo con los tricodermios entrelazados. La basidia mide $32.5-38 \times 10-13$ micras; amarillenta en $\mathrm{KOH}$. 
Habit. Formas gregarias, en bosques de coníferas.

Localidad. Sierra de Las Cruces.

Venenoso. Hay quienes aseguran que se puede comer.

Nombre vulgar. Galambo.

Observación. El píleo en los ejemplares jóvenes mexicanos es de color moreno obscuro, carácter un poco raro en comparación con ejemplares de Inglaterra y del Estado de Michigan (EE. UU.), que presentan el mismo color, tanto er las formas jóvenes como en las maduras; pero el tamaño de las esporas es muy similar; ejemplares de Inglaterra de 12-17 x 5.5-7 micras; ejemplares de Michigan, de 12-16 x 5.5-7 micras.

\section{Boletus placidus Bonarden}

Píleo de 3-6 cm. de ancho, convexo, de superficie viscosa o glutinosa, blanda al tacto, de color amarillo claro que pasa a ocre obscuro con la edad. Carne blanca o amarillo pálida, gusto no especial.

Tubos de 5-8 mm., de color amarillo pálido, decurrentes; al contacto del aire no cambian de color. Poros pequeños y redondos.

Estípite de 3-6 de largo, por 8-12 mm. de grueso en el ápice y 1.5$1.7 \mathrm{~cm}$. de grueso en la base, de color encarnado.

Esporas de 7-10 x 2.5-3.5 micras, de color amarillo pálido en $\mathrm{KOH}$; lisas.

Habitat. Hongo gregario, en bosques de coníferas.

Localidad. Desierto de los Leones.

\section{Boletus porphyrosporus $\mathrm{Fr}$ :}

Píleo de 4-4.5 cm. de ancho, convexo, se vuelve plano con la edad; superficie seca y lisa, de color moreno claro o moreno obscuro. Carne de $6 \mathrm{~mm}$. de ancho, blanda y esponjosa, blanquizca, que al contacto del aire toma un color azul; sabor no especial, olor ocre.

Tubos de $9 \mathrm{~mm}$. de profundidad, deprimidos alrededor del estípite, de color grisáceo moreno; al tocarlos, toman un color azul verdoso. Poros pequeños, redondos, de color moreno.

Estípite de $8.5 \mathrm{~cm}$. de largo por $1.7 \mathrm{~cm}$. de grueso en el ápice y $2.5 \mathrm{~cm}$. de grueso en la base, sólido, de color moreno obscuro en la base y moreno claro en el ápice; ligeramente reticulado cerca del ápice.

Esporas de $14-18 \times 6-7.5$ micras, de color moreno muy pálido en $\mathrm{KOH}$, lisas, paredes gruesas. Basidios de $45-50$ x 9-14 micras, color mo- 


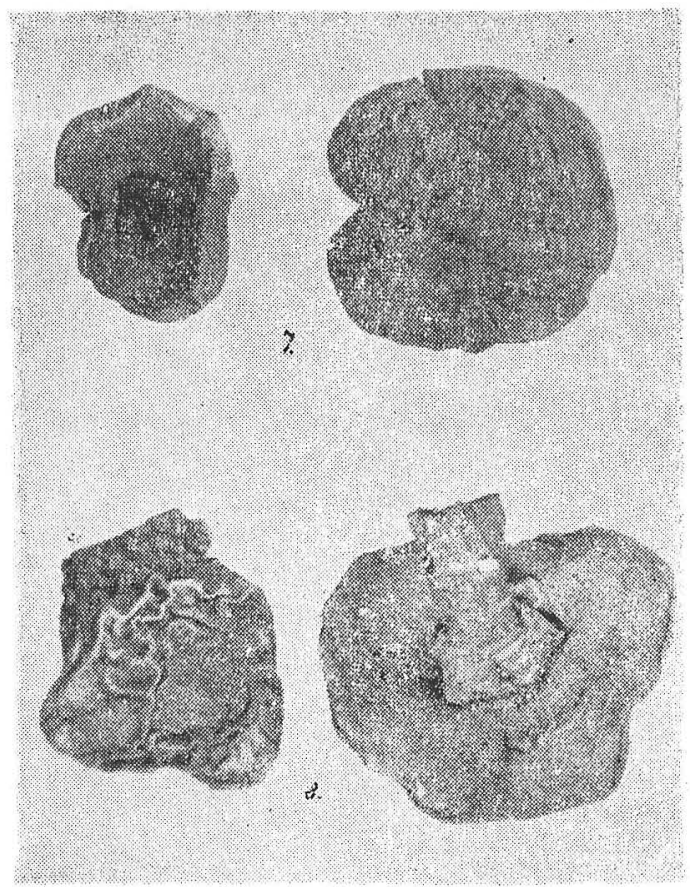

7. BOLETUS PORPHYROSPORUS, vista ventral dorsal; 8 BOLETUS PULVERULENTUS, vista dorsal y ventral.

reno tabaco en $\mathrm{KOH}$. La cutícula del píleo con tricodermios bien desarrollados.

Habitat. Gregario, en bosque de coníferas.

Localidad. Sierra de Las Cruces.

\section{Boletus pulverulentus Opat.}

Píleo de 2-9 cm. de ancho, convexo cuando joven, volviéndose plano con la edad; superficie seca, de color moreno obscuro. Carne de 4-5 mm. de grosor, color amarillo claro, blanda, esponjosa y gruesa; al contacto del aire toma un color azul intenso; sabor y olor no especiales.

Tubos de color amarillo limón, adnatos, ligeramente decurrentes, no se deprimen con la edad. Poros redondos, a veces angulares, alrededor de uno por mm.; toman un color azul intenso al contacto del aire. 
Estípite de 5-9 cm. de largo por 1.5-4 cm. de grueso, carnoso, de color moreno obscuro y leonado en la base.

Esporas de 10-15 x 4-5 micras, de color moreno oliváceo; aparecen estrechas al ser vistas ventralmente; tiene paredes lisas.

Habit. Gregario, se encontró en bosque de coníferas, en suelo con musgo.

Localidad. Sierra de Las Cruces.

Observación. Las esporas encontradas en los ejemplares de Michigan (EE. UU.) fueron de tamaño muy similar al de los ejemplares mexicanos: 10-14 x 4-5 micras.

\section{Boletus subtomentosus Snell.}

Píleo de 2-5 cm. de ancho, convexo, volviéndose ligeramente plano; superficie seca, granular cuando se observa con lente de aumento; color

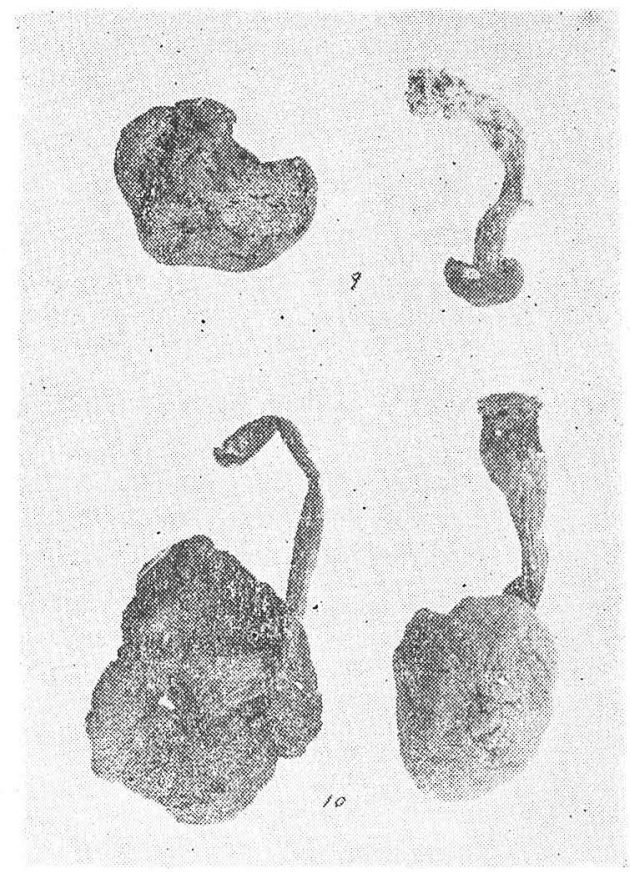

9, BOLETUS SUBTOMENTOSUS, vista dorsal y ventral; BOLETUS TURBINATUS, vista dorsal y ventral. 
moreno obscuro. Carne de $1.5 \mathrm{~cm}$. de grosor y de color amarillo pálido; al contacto con el aire no cambia de color; olor y sabor no especiales.

Tubos de $7 \mathrm{~mm}$. de profundidad, decurrentes, color amarillo olivo; no cambian de color cuando se cortan o tocan. Poros angulares e irregulares.

Estípite de 3.5-4.5 $\mathrm{cm}$. de longitud y de 5-7 $\mathrm{mm}$. de espesor, más o menos de igual grosor en toda su longitud, de color amarillo o blanquizco; sólido; presenta una ligera reticulación cerca del ápice.

Esporas de 11-14 x 4-4.5 micras, subfusiformes, lisas, amarillas ocráceas en KOH. Basidios de 24-30 x 7-10 micras, amarillo en $\mathrm{KOH}$. Cutícula del píleo con tricodermios de color amarillo en $\mathrm{KOH}$.

Habitat. Solitario o gregario; se encuentra bajo los pinos.

Localidad. Desierto de los Leones (cerro de San Miguel a 3,400 m.). Comestible.

Observación. El tamaño de las esporas de los ejemplares mexicanos difiere muy poco del de los ejemplares estudiados en Inglaterra (12$16 \times 4.5-5.5$ micras) y del de Pellston, EE. UU. (12-16 x 4-4.5 micras).

\section{Boletus turbinatus Snell.}

Píleo de 2.5-4 cm. de ancho, moreno, con forfuraciones adnatas de color moreno obscuro, convexo cuando joven y aplanándose cuando madura; superficie seca. Carne amarillenta, cuando se corta toma un color azul intenso.

Tubos de color amarillo; su longitud es de 3-8 mm.; adnatos, poros de color amarillo leonado.

Estípite de 3-6 cm. de longitud por 7-9.5 mm. de espesor, más o menos fusiforme, moreno claro en la parte superior y moreno rojizo en las partes media e inferior.

Esporas de 11-16 x 5-6 micras, presentan un color amarillo ámbar en $\mathrm{KOH}$; paredes lisas pero gruesas; presentan una forma elíptica, más ancha en la parte distal y más delgada en la parte proximal. Presentan una peculiaridad propia de la especie que consiste en tener un poro germinativo incompleto.

Habitat. Gregario o solitario; abundante en bosques de coníferas, en tierra cubierta por musgo.

Localidad. Desierto de los Leones (cerro de San Miguel a 3,400 m.) y cañada de Contreras (cerro de la Coconetla), aproximadamente a la misma altitud anotada antes. 


\section{Boletus sp.}

Píleo de $5 \mathrm{~cm}$. de ancho, convexo; superficie seca, lisa; color moreno obscuro. Carne de $6 \mathrm{~mm}$. de grosor, firme, color amarillo pálido, al contacto del aire no cambia de color, olor y sabor no especiales.

Tubos deprimidos alrededor del estípite, de color amarillo verdoso, de $6 \mathrm{~mm}$. de profundidad. Poros de color rojo ladrillo.

Estípite de $9 \mathrm{~cm}$. de longitud por $1.3 \mathrm{~cm}$. de ancho en el ápice y de 1.5-2 cm. de ancho en la base, sólido, de color moreno con estrías rojizas en la base y amarillo leonado en el ápice.

Esporas de 15-20 (22) x 5-6.6 micras, lisas de paredes gruesas; en KOH presentan un color ocre pálido. Basidio de 23-30 x 9-10 micras.

Habitat. Solitario, en bosques de pinos.

Localidad. Sierra de Las Cruces.

Observación. La descripción está basada en un solo ejemplar.

\section{Boletus sp.}

Píleo de 4-4.5 cm. de ancho, convexo, volviéndose ligeramente plano con la edad; superficie seca, de color moreno obscuro y con forfuraciones adnatas. Carne de $4 \mathrm{~mm}$. de grosor, de color blanco amarillento; al contacto del aire no cambia de color.

Tubos de $9 \mathrm{~mm}$. de profundidad, decurrentes, de color amarillo y que cuando se tocan o cortan no cambian de color; en los dos ejemplares encontrados los poros son grandes, presentándose angulares y amarillos en el ejemplar joven, y morenos ligeramente anaranjados en el ejemplar maduro.

Estípite de $3-3.5 \mathrm{~cm}$. de largo, delgado, de igual grosor en toda su longitud, color moreno muy obscuro en la base y moreno claro o amarillo leonado en el ápice.

Esporas de 13-17 x 5.5-6 micras en $\mathrm{KOH}$; subfusiformes, lisas, de paredes gruesas. Basidios de 20-25 x 9-11 micras.

Habitat. Solitario, en bosques de coníferas.

Localidad. Sierra de Las Cruces.

Observación. Esta especie presenta caracteres muy similares a los de Boletus subtomentosus; difiere en el grosor de la carne, en el tamaño de los poros, en la longitud y grosor de las esporas y en el tamaño de los basidios. No se ha podido consignar como especie nueva debido a que sólo se colectaron dos ejemplares, uno joven y uno maduro.

RESUMEN.-Del material encontrado se pudieron diferencial doce especies, de las cuales se ha hecho la correspondiente descripción; de ellas, diez han sido clasificadas y las otras dos se han dejado sin clasificar por el momento, debido a que únicamente se encontró un ejemplar de una de ellas y dos de la otra; por las características anotadas en las descripciones, puede sospecharse que los ejemplares mencionados corresponden a especies nuevas. 


\section{BIBLIOGRAFIA}

Bataille, F. "Les reactions macrochimiques chez les champignons". Lechevalier. París, 1948.

Canara, F. "Funghi e Tartufi". Hoepli. Milano. 1943.

Coker, W. C. and Beer, A. H. "The Boletaceae of North Carolina". The University of North Carolina Press. 1943.

Gilbert, E. J. "Les Bolets". pp. 1-225. Librairie E. le Francois. Paris. 1931.

Herter, G. "Champignons comestibles". Lechevalier. Paris. 1951.

Kallenbach, F. "Die Röhrlinge in Pilze Mitteleuropas". Vol. 1. (Boletaceae). Leipzig. 1926.

Kühner, R. et Tomagnesi, H. "Flore analytique des Champignons superieurs". pp. 33-46. Masson et Cie., editeurs. 1953.

Maublanc, A. "Les Champignons comestibles et vénéneus". Lechevalier. Vol. II. París. 1952.

Murrill, W. A. "The Boletaceae of North America". I and II. Mycologia 1:4-18, 140-160. 1909.

Murrill, W. A. "Edible and poisonous Mushrooms". New York. 1916. Singer, R. "The Boletoideae of Florida". I. Farlowia 2:97-141. 1945. II. Farlowia $2: 223-303.1945$.

Singer, R. "The American Midland Naturalist. Vol. 37. 1947.

Snell, W. H. "Notes on Boletes". IV. Mycologia 28:13-23. 1936. V. Mycologia 28:463-475. 1936.

Snell, W. H. "Tentative keys to the Boletaceae of the United States and Canada". Publ. No. 1. Rhode Island Bot. Cl. 1:25. 1936.

Snell, W. H. "The Genera of Boletaceae". VI. Mycologia 33: 415-423. 1941.

Snell, W. H. and Dick, E. A. "Notes on Boletes". VI. Mycologia 33:2327. 1941.

Smith, A. H. "Mushrooms in their natural habitats". Publ. Sawyer's Inc. Portland, Ore. 1949.

Thiers, H. D. "The Agaricaceae of the pine belt and adjacent areas in Eastern Texas". (Boletaceae). pp. 25-59. University of Michigan. 1956.

Thomas W. H. "Field book of common mushrooms". Putnam's Sons. New York-London. 1948. 